\begin{tabular}{|c|l|}
\hline Title & Probing neutron-skin thickness with total reaction cross sections \\
\hline Author(s) & Horiuchi, W ataru; Suzuki, Y.; Inakura, T. \\
\hline Citation & $\begin{array}{l}\text { Physical Review C, 89(1), 011601 } \\
\text { https://doi.org/L0.1103/PhysRevC.89.011601 }\end{array}$ \\
\hline Issue Date & 20141-22 \\
\hline Doc URL & http://hdl.handle.net/2115/55350 \\
\hline Rights & @2014 A merican Physical Society \\
\hline Type & article \\
\hline File Information & PhysRevC.89.011601.pdf \\
\hline
\end{tabular}

Instructions for use 


\title{
Probing neutron-skin thickness with total reaction cross sections
}

\author{
W. Horiuchi, ${ }^{1}$ Y. Suzuki, ${ }^{2,3}$ and T. Inakura ${ }^{4}$ \\ ${ }^{1}$ Department of Physics, Hokkaido University, Sapporo 060-0810, Japan \\ ${ }^{2}$ Department of Physics, Niigata University, Niigata 950-2181, Japan \\ ${ }^{3}$ RIKEN Nishina Center, Wako 351-0198, Japan \\ ${ }^{4}$ Department of Physics, Graduate School of Science, Chiba University, Chiba, 263-8522, Japan
}

(Received 29 November 2013; published 22 January 2014)

\begin{abstract}
We analyze total reaction cross sections, $\sigma_{R}$, to explore their sensitivity to the neutron-skin thickness of nuclei. We cover 91 nuclei of $\mathrm{O}, \mathrm{Ne}, \mathrm{Mg}, \mathrm{Si}, \mathrm{S}, \mathrm{Ca}$, and $\mathrm{Ni}$ isotopes. The cross sections are calculated in the Glauber theory using the density distributions obtained with the Skyrme-Hartree-Fock method in three-dimensional coordinate space. Defining a reaction radius, $a_{R}=\sqrt{\sigma_{R} / \pi}$, to characterize the nuclear size and target (proton or ${ }^{12} \mathrm{C}$ ) dependence, we find an empirical formula for expressing $a_{R}$ with the point matter radius and the skin thickness, and assess two practical ways of determining the skin thickness from proton-nucleus $\sigma_{R}$ values measured at different energies or from $\sigma_{R}$ values measured for different targets.
\end{abstract}

DOI: 10.1103/PhysRevC.89.011601

PACS number(s): 25.60.Dz, 27.30.+t, 27.40.+z, 27.50.+e

A systematic study of nuclear size properties tells us the saturation property of atomic nuclei. Recently the nuclear isovector size property, that is, neutron-skin thickness, has attracted much interest. The knowledge of skin thickness gives more insight into the properties of neutron-rich nuclei and neutron stars, and the equation of state (EOS) of asymmetric nuclear matter. For example, it is pointed out in Refs. [1-4] that the skin thickness of finite nuclei constrains the symmetry energy and the slope parameter $L$ of the pure neutron matter EOS at the saturation density, which is one of the key ingredients for the two-solar-mass neutron star problem [5].

The skin thickness has also been studied experimentally. The parity-violating elastic electron scattering [6] has been performed to determine the skin thickness of ${ }^{208} \mathrm{~Pb}$, yielding $0.33_{-0.18}^{+0.16} \mathrm{fm}$. Further measurement is planned to get more precise data for ${ }^{208} \mathrm{~Pb}$ [7]. Since the neutron radius is difficult to probe, measurements of the skin thickness are still not as precise as those of the proton radius, which is extracted from the charge distribution obtained by an electron scattering.

A hadronic probe is also useful to study the size properties of nuclei. Proton elastic scattering measurements at $295 \mathrm{MeV}$ have been done to probe the nuclear distributions of heavy stable targets, $\mathrm{Sn}[8]$ and $\mathrm{Pb}[9]$ isotopes. Electric dipole response has been measured for ${ }^{208} \mathrm{~Pb}$ using a $\left(p, p^{\prime}\right)$ reaction and the skin thickness is evaluated as $0.156_{-0.021}^{+0.025} \mathrm{fm}$ by making use of the dipole polarizability of ${ }^{208} \mathrm{~Pb}$ [10]. However, it is difficult to extend such measurements to unstable nuclei because of their short lifetimes.

Total reaction or interaction cross sections for unstable nuclei are more easily and accurately measured as long as they are produced sufficiently. Recent radioactive ion beam facilities allow us to measure precise total reaction cross sections for neutron-rich $\mathrm{Ne}$ and $\mathrm{Mg}$ isotopes on ${ }^{12} \mathrm{C}$ target [11-13]. The total reaction cross section on ${ }^{12} \mathrm{C}$ target primarily probes the matter radius, and therefore we need to know neutron or proton radii to determine the skin thickness. The charge radii of unstable nuclei is made available by isotope shift measurements [14-16]. A combination of the deduced matter and proton radii from the different experiments gives us information on the skin thickness. The isotope shift measurement is, however, at present possible only for some limited cases; moreover, deducing the charge radius from the measurement calls for extensive evaluations for various corrections. The measurement of the charge-changing cross section may be an alternative for probing the proton radius, but that cross section does not always probe the proton radius directly, and thus one needs some model-dependent corrections to extract the proton radius [17].

The purpose of this study is to discuss the possibility of using the total reaction cross sections to extract the skin thickness. Recalling the fact that the neutron-proton total cross section is larger than that of the proton-proton below the incident energy of $550 \mathrm{MeV}$, we expect a proton target to probe more sensitively the neutron distribution in the tail region than a ${ }^{12} \mathrm{C}$ target [18]. Based on the Glauber formalism [19], we systematically analyze the total reaction cross sections for many nuclei with mass numbers $A=14-86$ of $\mathrm{O}, \mathrm{Ne}$, $\mathrm{Mg}, \mathrm{Si}, \mathrm{S}, \mathrm{Ca}$, and $\mathrm{Ni}$ isotopes. The wave functions of those nuclei are generated by the Skyrme-Hartree-Fock method on three-dimensional coordinate space. The analysis of the cross sections enables us to propose possible ways to extract the skin thickness through the energy and target dependence of the total reaction cross sections.

The total reaction cross section is calculated by

$$
\sigma_{R}=\int d \boldsymbol{b}\left(1-\left|e^{i \chi(\boldsymbol{b})}\right|^{2}\right)
$$

where $\chi(\boldsymbol{b})$ is the phase-shift function for the elastic scattering of a projectile nucleus $(P)$ and a target nucleus $(T)$ and the integration is done over the impact parameter $\boldsymbol{b}$. The phase-shift function is defined by a multiple integration with the ground state wave functions of the projectile and target and its evaluation may be performed with a Monte Carlo technique as was done in Ref. [21]. Here we use the so-called optical limit approximation (OLA), which requires only the one-body 
densities of the projectile and target, $\rho_{P}$ and $\rho_{T}$, respectively,

$$
\begin{aligned}
e^{i \chi(\boldsymbol{b})}= & \exp \left[-\iint d \boldsymbol{r}^{P} d \boldsymbol{r}^{T} \rho_{P}\left(\boldsymbol{r}^{P}\right) \rho_{T}\left(\boldsymbol{r}^{T}\right)\right. \\
& \left.\times \Gamma_{N N}\left(\boldsymbol{s}^{P}-\boldsymbol{s}^{T}+\boldsymbol{b}\right)\right]
\end{aligned}
$$

where $\boldsymbol{s}^{P}\left(\boldsymbol{s}^{T}\right)$ is the transverse component of $\boldsymbol{r}^{P}\left(\boldsymbol{r}^{T}\right)$ perpendicular to the beam direction, and $\Gamma_{N N}$ is the nucleonnucleon $(N N)$ profile function describing the $N N$ collision at incident energy $E$. The profile function is different between proton-proton $(p p)$ and proton-neutron $(p n)$. The profile function for neutron-neutron is taken the same as $p p$. The integration in Eq. (2) is carried out using the proton and neutron densities of the projectile and target.

We use a usual parametrization for $\Gamma_{N N}$,

$$
\Gamma_{N N}(\boldsymbol{b})=\frac{1-i \alpha_{N N}}{4 \pi \beta_{N N}} \sigma_{N N}^{\text {tot }} \exp \left[-\frac{\boldsymbol{b}^{2}}{2 \beta_{N N}}\right],
$$

where $\alpha_{N N}$ is the ratio of the real to the imaginary part of the $N N$ scattering amplitude in the forward angle, $\beta_{N N}$ is the slope parameter of the $N N$ elastic scattering differential cross section, and $\sigma_{N N}^{\text {tot }}$ is the total cross section of the $N N$ scattering. They are tabulated in Ref. [18] for a wide range of energies. Though it misses some higher-order terms of $\Gamma_{N N}$, the OLA describes the proton-nucleus scattering satisfactorily [21]. However, the OLA for nucleus-nucleus collisions tends to predict larger cross sections than measurement [22], and we employ another expression called the nucleon target formalism in the Glauber theory (NTG) [20]. The NTG requires the same inputs as the OLA and reproduces $\sigma_{R}$ fairly well. For example, $\sigma_{R}$ of ${ }^{12} \mathrm{C}+{ }^{12} \mathrm{C}$ calculated with NTG are improved very much in a wide energy range [22]. The power of NTG is also confirmed in applications to ${ }^{22} \mathrm{C}$ [23], oxygen isotopes [24], and light neutron-rich nuclei $[25,26]$. In this paper we employ the OLA for the proton target and the NTG for the ${ }^{12} \mathrm{C}$ target.

For projectiles with large $Z$, the Coulomb force contributes to $\sigma_{R}$ via Coulomb breakup. Its effect may be taken into account by, e.g., the Coulomb corrected eikonal approximation [27-29]. Since $Z$ involved in the present calculation is not very large, the Coulomb interaction between the projectile and target is ignored for the sake of simplicity.

We perform the Skyrme-Hartree-Fock (HF) calculation for the density distribution of a variety of projectiles. The ground state is obtained by minimizing the energy density functional [30]. Every single-particle wave function is represented in the three-dimensional grid points with the mesh size of $0.8 \mathrm{fm}$. All the grid points inside the sphere of radius of $20 \mathrm{fm}$ are adopted in the model space. The ground state is constructed by the imaginary-time method [31]. The angle-averaged intrinsic one-body densities are used as the one-body densities required for the Glauber calculation. For more detail, see Ref. [26].

We consider light to medium mass even-even nuclei with $Z=8-16,20$, and 28 , covering both proton- and neutron-rich regions, that is, ${ }^{14-24} \mathrm{O},{ }^{18-34} \mathrm{Ne},{ }^{20-40} \mathrm{Mg},{ }^{22-46} \mathrm{Si},{ }^{26-50} \mathrm{~S}$, ${ }^{34-70} \mathrm{Ca}$, and ${ }^{48-86} \mathrm{Ni}$. Two Skyrme parameter sets, SkM* [32] and SLy4 [33], are employed. The SkM* functional is known to well account for the properties of the nuclear deformation, while the SLy4 is superior to SkM* in reproducing the properties of neutron-rich nuclei. In Ref. [26] we study light neutron-rich nuclei with even proton numbers $Z=8-16$ and discuss the deformation effects on $\sigma_{R}$ for the ${ }^{12} \mathrm{C}$ target. The two interactions give a significant difference in $\sigma_{R}$ due to the nuclear deformation [26].

As is well known, $\sigma_{R}$ carries information on the nuclear size. It is convenient to define a "reaction radius" $a_{R}$ of the nucleus-nucleus collision at incident energy $E$ by

$$
a_{R}(N, Z, E, T)=\sqrt{\sigma_{R}(N, Z, E, T) / \pi},
$$

where $N$ and $Z$ are the neutron and proton numbers of the projectile, and $T$ stands for the target, either proton or ${ }^{12} \mathrm{C}$. In what follows we omit $T$ in most cases and mean by $E$ the projectile's incident energy per nucleon. The reaction radius depends on $E$ through the energy dependence of the profile function (3). The $p n$ and $p p$ total cross sections, $\sigma_{p n}^{\text {tot }}$ and $\sigma_{p p}^{\text {tot }}$, behave differently as follows [34]. At $E=100 \mathrm{MeV}, \sigma_{p n}^{\text {tot }}$ is about three times larger than $\sigma_{p p}^{\text {tot }}$. The difference between them gets smaller as the energy increases and vanishes at about $550 \mathrm{MeV}$, and beyond $800 \mathrm{MeV} \sigma_{p p}^{\text {tot }}$ exceeds $\sigma_{p n}^{\text {tot }}$. The above energy dependence of the basic inputs of $N N$ data is best reflected in $\sigma_{R}$ for the proton target, whereas it is averaged out in the case of the ${ }^{12} \mathrm{C}$ target. Thus we expect the proton target to be advantageous for probing the neutron-skin part.

We define the point matter root-mean-square (rms) radius $r_{m}(N, Z)$ and the skin thickness $\delta(N, Z)$ by

$$
\begin{aligned}
r_{m}(N, Z) & =\sqrt{\frac{Z}{A} r_{p}^{2}(N, Z)+\frac{N}{A} r_{n}^{2}(N, Z)}, \\
\delta(N, Z) & =r_{n}(N, Z)-r_{p}(N, Z),
\end{aligned}
$$

where $r_{p}(N, Z)$ and $r_{n}(N, Z)$ are point proton and point neutron rms radii, respectively. Figure 1 displays the reaction radii of the 91 nuclei as a function of $r_{m}(N, Z)$. As expected, $a_{R}$ shows a linear dependence on $r_{m}(N, Z)$ at any incident energies, which is the basis of extracting the matter radius from $\sigma_{R}$. A closer look at the proton case shows, however, somewhat

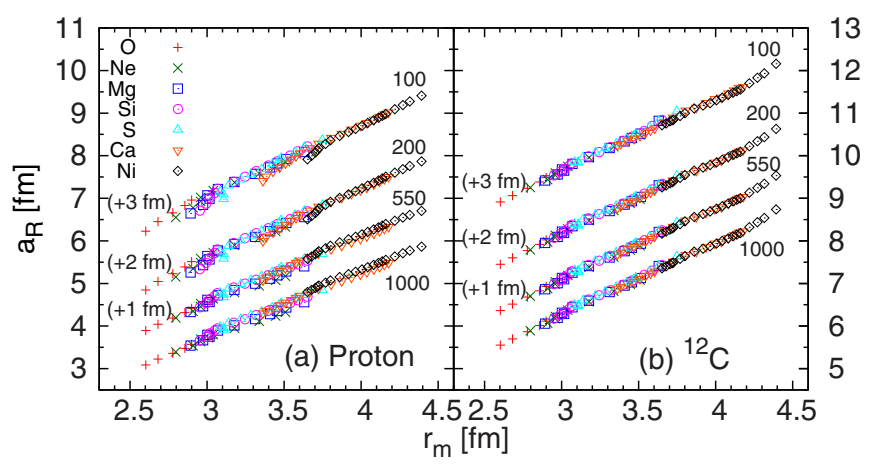

FIG. 1. (Color online) Reaction radii vs point matter rms radii for $\mathrm{O}, \mathrm{Ne}, \mathrm{Mg}, \mathrm{Si}, \mathrm{S}, \mathrm{Ca}$, and $\mathrm{Ni}$ isotopes on (a) proton and (b) ${ }^{12} \mathrm{C}$ targets at incident energies of $100,200,550$, and $1000 \mathrm{MeV}$. The SkM* interaction is used. The reaction radii of 550, 200, and $100 \mathrm{MeV}$ are added by 1,2 , and $3 \mathrm{fm}$, respectively, for the sake of illustration. 


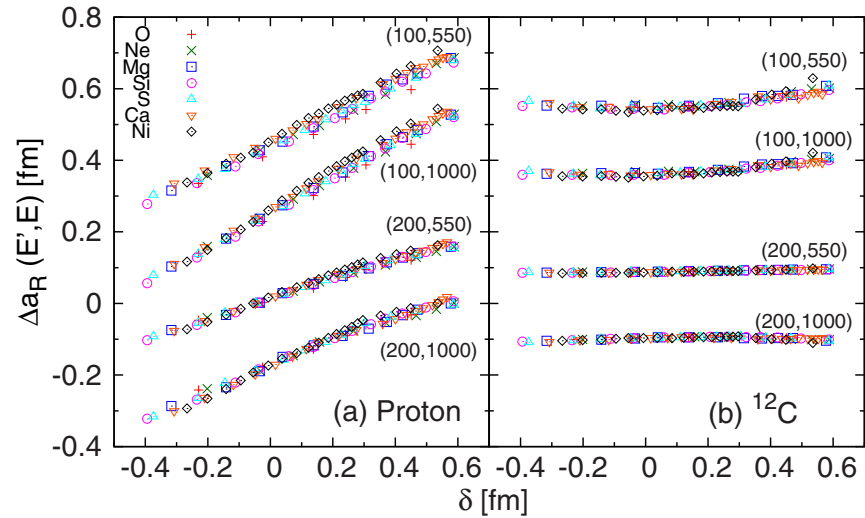

FIG. 2. (Color online) Difference of the reaction radii at two incident energies $\left(E^{\prime}, E\right)$ in $\mathrm{MeV}$ as a function of the skin thickness for $\mathrm{O}, \mathrm{Ne}, \mathrm{Mg}, \mathrm{Si}, \mathrm{S}, \mathrm{Ca}$, and $\mathrm{Ni}$ isotopes on (a) proton and (b) ${ }^{12} \mathrm{C}$ targets. The $\mathrm{SkM}^{*}$ interaction is used.

scattered distributions along the straight lines. This indicates that the reaction radii for the proton target scatter depending on the detail of the neutron and proton density profiles even though $r_{m}(N, Z)$ values are the same, namely, the reaction radii for the proton target carry some information on the skin thickness. The same conclusion is drawn with SLy4 as well.

To substantiate the above statement on probing the neutronproton density profiles, we consider the difference of the reaction radii at two incident energies

$$
\Delta a_{R}\left(N, Z, E^{\prime}, E\right)=a_{R}\left(N, Z, E^{\prime}\right)-a_{R}(N, Z, E) .
$$

Figure 2 plots $\Delta a_{R}$ as a function of $\delta(N, Z)$. Four different sets of energies are chosen. The isotope dependence of $\Delta a_{R}$ is weak for both targets, and in each set $\Delta a_{R}$ for all the nuclei tend to approximately follow a straight line. For proton target, the $\Delta a_{R}$ vs $\delta(N, Z)$ diagram exhibits strong correlation as revealed by a nonzero slope, that is, $\Delta a_{R}$ can be a quantity sensitive to the skin thickness. The steepest slope among the four sets is obtained for $\left(E^{\prime}, E\right)=(100,1000) \mathrm{MeV}$, which is easily understood from the energy dependence of $\sigma_{p n}^{\text {tot }}$ and $\sigma_{p p}^{\text {tot }}$. $\Delta a_{R}$ values for the ${ }^{12} \mathrm{C}$ target show a flat behavior, or the slope is almost zero, which is a consequence of the fact that the $p n$ and $p p$ profile functions are averaged because of the equal numbers of protons and neutrons in ${ }^{12} \mathrm{C}$. Thus the ${ }^{12} \mathrm{C}$ target is never sensitive to the skin thickness.

We confirm that the above observation holds in the SLy4 case as well, namely, the $\Delta a_{R}$ vs $\delta(N, Z)$ diagram obtained with the SLy4 interaction is almost the same as Fig. 2 obtained with $\mathrm{SkM}^{*}$. It appears that this is never trivial because the $\mathrm{SkM}^{*}$ and SLy4 interactions predict different density profiles as shown in Fig. 3, which compares $r_{m}(N, Z)$ and $\delta(N, Z)$ values of $\mathrm{Ne}, \mathrm{Mg}, \mathrm{Si}$, and $\mathrm{S}$ isotopes between the two sets. As discussed in Ref. [26], the matter radii of the $\mathrm{Ne}$ and $\mathrm{Mg}$ isotopes are correlated with their deformations that strongly depend on the Skyrme interactions. In fact we see the interaction dependence of $\sigma_{R}$ or $a_{R}$ in particular in the $Z=10-16$ region as shown in Fig. 3, whereas very similar results are obtained for $\mathrm{O}, \mathrm{Ca}$, and $\mathrm{Ni}$ isotopes. Despite this difference, the two Skyrme interactions give virtually the same

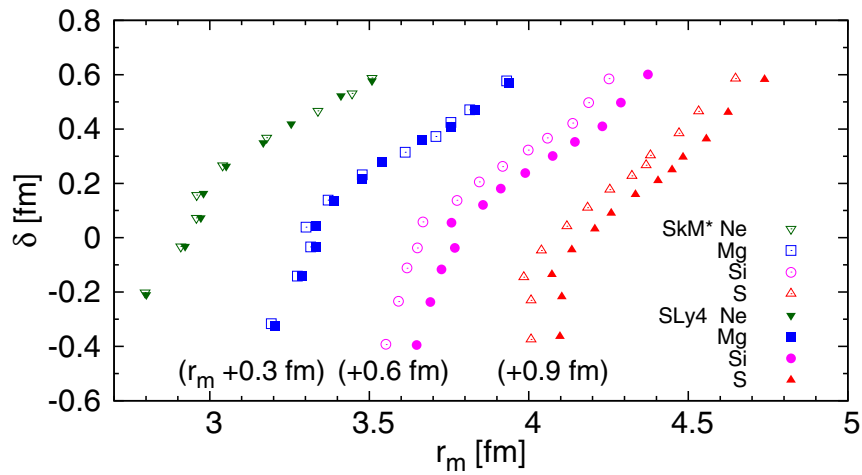

FIG. 3. (Color online) Comparison of the skin thickness and point matter rms radius of $\mathrm{Ne}, \mathrm{Mg}, \mathrm{Si}$, and $\mathrm{S}$ isotopes calculated with the $\mathrm{SkM}^{*}$ and SLy4 interactions. The radii of $\mathrm{Mg}, \mathrm{Si}$, and $\mathrm{S}$ isotopes are added by $0.3,0.6$, and $0.9 \mathrm{fm}$, respectively, for the sake of illustration.

$\Delta a_{R}$ vs $\delta(N, Z)$ diagram. Thus $\Delta a_{R}$ is sensitive to neither the nuclear shape nor the density distribution, but it is sensitive to the skin thickness.

The approximate linear dependence of both $a_{R}$ on $r_{m}(N, Z)$ and $\Delta a_{R}$ on $\delta(N, Z)$ suggests the following ansatz:

$$
a_{R}(N, Z, E)=\alpha(E) r_{m}(N, Z)+\beta(E) \delta(N, Z)+\gamma(E),
$$

where $\alpha(E), \beta(E)$, and $\gamma(E)$ depend on the target as well. Those coefficients are determined by minimizing the mean square deviation, $\chi^{2}(E)=\sum_{N, Z}\left[a_{R}^{\mathrm{HF}}(N, Z, E)-\right.$ $\left.a_{R}^{\mathrm{Fit}}(N, Z, E)\right]^{2} / \mathcal{N}$, where $a_{R}^{\mathrm{HF}}$ and $a_{R}^{\mathrm{Fit}}$ are $a_{R}$ values calculated with the HF densities and Eq. (7), respectively, and $\mathcal{N}=91$ is the number of data points. We cover 17 incident energies from 40 to $1000 \mathrm{MeV}$. It may be questionable to apply the Glauber theory to such low energy as $E=40 \mathrm{MeV}$ for the proton target, but we include it for the sake of convenience. The square root of $\sum_{i} \chi^{2}\left(E_{i}\right) / 17$ for the proton target is found to be 0.052 $\mathrm{fm}$ for $\mathrm{SkM}^{*}$ and 0.049 for SLy4, which is approximately $1 \%$ of the mean $a_{R}$ value, 4.68 and $4.70 \mathrm{fm}$, of all the nuclei at 17 energy points, respectively. As displayed in Fig. 4, the resulting coefficients show "universality," that is, very weak dependence on the Skyrme interactions, especially in the proton case. It should be stressed that Eq. (7) is valid for all the nuclei considered in this paper. We tried another ansatz by replacing $\delta(N, Z)$ with $\delta(N, Z) / r_{m}(N, Z)$, but the result was worse.

Both $\alpha(E)$ and $\gamma(E)$ weakly depend on $E$ above $100 \mathrm{MeV}$. The term with $\alpha(E)$ is a major contributor to $a_{R}$ among the three terms, and its weak energy dependence of less than a few $\%$ for both proton and ${ }^{12} \mathrm{C}$ targets explains the linearity of $a_{R}$ on $r_{m}(N, Z)$ with almost equal slopes as displayed in Fig. 1. The energy dependence of $\beta(E)$ is strong for the proton target. This is responsible for the scattered distributions of the reaction radii for the proton case as noted in Fig. 1. It is easy to understand that the linear dependence of $\Delta a_{R}$ on $\delta(N, Z)$ shows up in the proton case but almost disappears in the ${ }^{12} \mathrm{C}$ target as the main $\alpha(E)$ terms are canceled out. In this way the proton target probes the skin thickness sensitively but the ${ }^{12} \mathrm{C}$ target does not. The energy dependence of $\gamma(E)$ is similar between proton and ${ }^{12} \mathrm{C}$ targets. The latter is obtained 


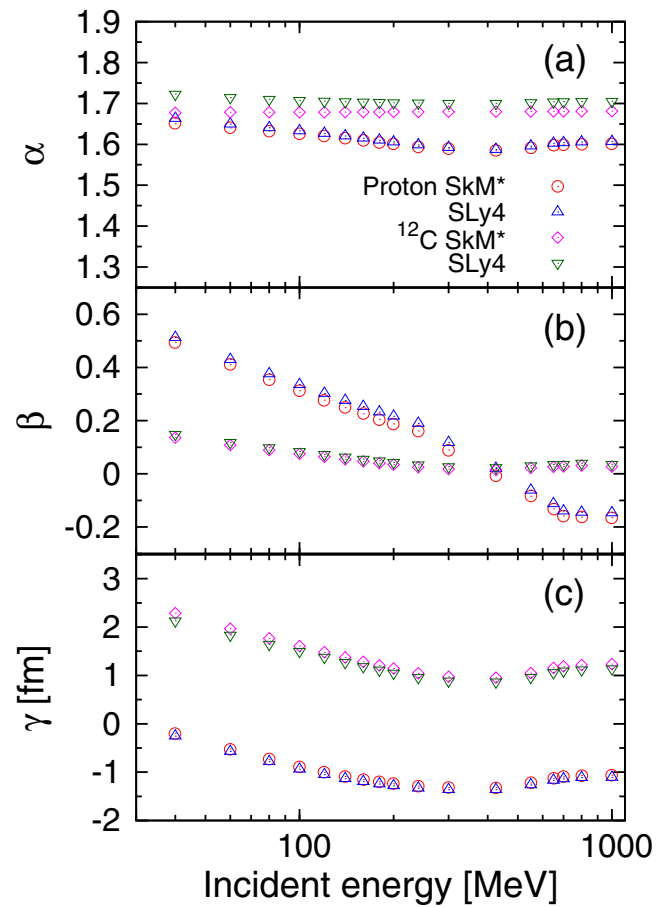

FIG. 4. (Color online) Coefficients of reaction radius, (a) $\alpha(E)$, (b) $\beta(E)$, and (c) $\gamma(E)$ as a function of the projectile's incident energy per nucleon. The SkM* and SLy4 interactions are used.

by adding about $2.4 \mathrm{fm}$ to $\gamma(E)$ of the proton target, which is understandable considering that the reaction radius of the ${ }^{12} \mathrm{C}$ target includes not only the radius of the projectile but also that of ${ }^{12} \mathrm{C}$.

We have determined $\alpha(E), \beta(E)$, and $\gamma(E)$ using the results on the 91 nuclei obtained with the two Skyrme interactions. It will be interesting to further test the universal relation between $a_{R}$ and $r_{m}(N, Z)$ as well as $\delta(N, Z)$ in heavier nuclei using different Skyrme interactions.

Because Eq. (7) is found to be valid to good approximation, we can make use of it to know the skin thickness. Given that two $\sigma_{R}$ or $a_{R}$ values for a proton target are accurately measured at different energies, we can extract $r_{m}(N, Z)$ and $\delta(N, Z)$ values as follows. A crude estimate of those unknowns is to solve a simultaneous linear equation based on Eq. (7). Since precise values of $\alpha(E), \beta(E)$, and $\gamma(E)$ may actually be unknown, another way without recourse to them is preferable. We assume some model density distributions for protons and neutrons and calculate $\sigma_{R}$ values. If those cross sections agree with the measured cross sections simultaneously, $r_{m}(N, Z)$ and $\delta(N, Z)$ are calculated from the assumed distributions. Otherwise other density distributions are tested until the model distributions reproduce the measured cross sections. Here the model distribution may be, for instance, Fermi type. This assertion is based on the fact that $\sigma_{R}$ is not sensitive to the detail of the density distribution but is determined by $r_{m}(N, Z)$ and $\delta(N, Z)$. Conversely, two different density distributions predict virtually the same $\sigma_{R}$ value in so far as they give the same $r_{m}(N, Z)$ and $\delta(N, Z)$ values. To corroborate the statement, we compare in Table I $\sigma_{R}$ values of $p+{ }^{40} \mathrm{Si}$ calculated using Fermi distributions with different diffuseness parameters. The
TABLE I. $p+{ }^{40} \mathrm{Si}$ total reaction cross sections in $\mathrm{mb}$ at incident energy $E$ in $\mathrm{MeV}$ calculated with different Fermi distributions. Each of the distributions is specified by the proton and neutron diffuseness parameters in fm, $a_{p}$ and $a_{n}$, and the proton and neutron radius parameters are set to reproduce the matter radius $(3.46 \mathrm{fm})$ and skin thickness $(0.37 \mathrm{fm})$ of ${ }^{40} \mathrm{Si}$ obtained with the $\mathrm{SkM} * \mathrm{HF}$ calculation. See Fig. 5.

\begin{tabular}{lllllllllll}
\hline \hline$\left(a_{p}, a_{n}\right) \backslash E$ & 100 & 120 & 140 & 160 & 200 & 300 & 425 & 550 & 800 & 1000 \\
\hline$(0.5,0.5)$ & 742 & 700 & 667 & 642 & 607 & 564 & 550 & 575 & 615 & 619 \\
$(0.6,0.6)$ & 752 & 706 & 672 & 646 & 609 & 565 & 551 & 577 & 619 & 622 \\
$(0.7,0.7)$ & 759 & 711 & 675 & 648 & 609 & 564 & 549 & 576 & 621 & 623 \\
$(0.5,0.7)$ & 756 & 708 & 673 & 646 & 608 & 563 & 549 & 575 & 617 & 620 \\
$\mathrm{HF}\left(\mathrm{SkM}^{*}\right)$ & 747 & 703 & 670 & 644 & 608 & 565 & 551 & 576 & 617 & 620 \\
\hline \hline
\end{tabular}

proton and neutron radius parameters of each distribution is set to reproduce the $r_{m}(N, Z)$ and $\delta(N, Z)$ values of the $\mathrm{SkM}^{*}$ density. The tail parts of the densities are crucially important to determine $\sigma_{R}$. Though the shapes of the distributions are different as shown in Fig. 5, all the densities give $\sigma_{R}$ very close to the HF cross section at all the energies. Even at $100 \mathrm{MeV}$ the deviation from the HF result falls within at most $1.6 \%$, confirming our statement. For a practical measurement, the two energies are to be chosen from low and high energy regions, e.g., $E \lesssim 200$ and $E \gtrsim 550 \mathrm{MeV}$, to make use of the sensitivity to the skin thickness as shown in the energy dependence of $\beta(E)$, and the difference of the two cross sections has to be large enough to be distinguished beyond experimental uncertainties.

Another possible way is to measure $\sigma_{R}$ values on proton and ${ }^{12} \mathrm{C}$ targets. Assuming some model densities for protons and neutrons, $r_{m}(N, Z)$ and $\delta(N, Z)$ can also be determined by reproducing the $\sigma_{R}$ simultaneously. The incident energy for ${ }^{12} \mathrm{C}$ target may be chosen arbitrarily because the $a_{R}$ on ${ }^{12} \mathrm{C}$ target is only sensitive to $r_{m}(N, Z)$ as shown in Fig. 4. For a proton target, it is advantageous to choose low energy, say $E \lesssim 200$, for maximizing the sensitivity to $\delta(N, Z)$ as much as possible. Table II lists $\sigma_{R}$ on a ${ }^{12} \mathrm{C}$ target obtained with the different Fermi distributions. The deviation from the $\mathrm{HF}$ result is about $5 \%$ for all $E$, which is not as small

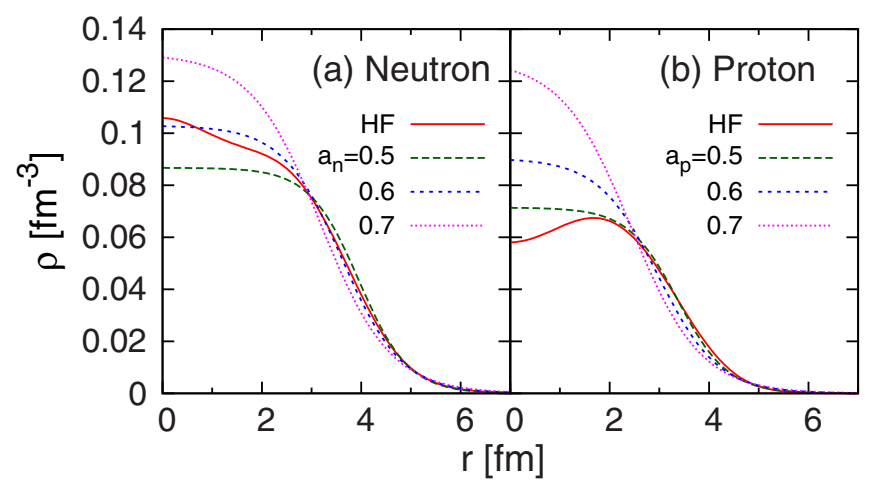

FIG. 5. (Color online) Matter, neutron, and proton densities of ${ }^{40} \mathrm{Si}$ of Fermi distributions with different sets of the diffuseness parameter. The HF densities with $\mathrm{SkM}^{*}$ are also plotted for comparison. 
TABLE II. Same as Table I but on ${ }^{12} \mathrm{C}$ target in units of $\mathrm{b}$.

\begin{tabular}{lccccccccc}
\hline$\left(a_{p}, a_{n}\right) \backslash E$ & 100 & 140 & 160 & 200 & 300 & 425 & 550 & 800 & 1000 \\
\hline$(0.5,0.5)$ & 1.73 & 1.62 & 1.58 & 1.52 & 1.45 & 1.44 & 1.48 & 1.55 & 1.56 \\
$(0.6,0.6)$ & 1.78 & 1.66 & 1.62 & 1.56 & 1.48 & 1.47 & 1.51 & 1.59 & 1.60 \\
$(0.7,0.7)$ & 1.84 & 1.71 & 1.67 & 1.60 & 1.52 & 1.51 & 1.55 & 1.64 & 1.65 \\
$(0.5,0.7)$ & 1.80 & 1.68 & 1.64 & 1.57 & 1.49 & 1.48 & 1.53 & 1.61 & 1.62 \\
$\mathrm{HF}_{\left(\mathrm{SkM}^{*}\right)}$ & 1.74 & 1.63 & 1.59 & 1.53 & 1.45 & 1.44 & 1.49 & 1.56 & 1.57 \\
\hline \hline
\end{tabular}

as that of a proton target. Because of this we must admit that constraining $r_{m}(N, Z)$ through $\sigma_{R}$ for the ${ }^{12} \mathrm{C}$ target contains some uncertainty. In Ref. [11], $\sigma_{R}$ for proton and ${ }^{12} \mathrm{C}$ targets are measured for neutron-rich $\mathrm{Mg}$ isotopes at $900 \mathrm{MeV}$, and $r_{m}(N, Z)$ of ${ }^{32-35} \mathrm{Mg}$ isotopes are determined within approximately $5 \%$ in the analysis with the Fermi density distributions. Unfortunately $\sigma_{R}$ on a proton target did not set a constraint on $\delta(N, Z)$ because the proton data contained large uncertainty and the profile function at $900 \mathrm{MeV}$ is insensitive to the neutron-skin thickness. It is necessary to reduce the experimental uncertainty of $\sigma_{R}$ on the proton target for a precise determination of $r_{m}(N, Z)$ and $\delta(N, Z)$.
In summary, to explore a sensitive probe to the skin thickness, we have made a systematic analysis of total reaction cross sections, $\sigma_{R}$, on proton and ${ }^{12} \mathrm{C}$ targets in the Glauber model. The Skyrme-Hartree-Fock method is applied to generate the densities of 91 even-even nuclei with $A=$ 14-86 from $Z=8-16,20$, and 28 elements. Two different interactions, $\mathrm{SkM}^{*}$ and SLy4, are employed to test the nuclear size properties.

We find a universal expression that linearly relates the reaction radius, $a_{R}=\sqrt{\sigma_{R} / \pi}$, to the point matter radius $r_{m}(N, Z)$ and skin thickness $\delta(N, Z)$. The proportional coefficients are determined as a function of the incident energy by analyzing $\sigma_{R}$ calculated from the Hartree-Fock densities. Among others, the coefficient proportional to $\delta(N, Z)$ in the case of a proton target exhibits remarkable energy dependence. This sensitivity of $a_{R}$ or $\sigma_{R}$ to the skin thickness has enabled us to assess a practical way of determining both $\delta(N, Z)$ and $r_{m}(N, Z)$ from the measurements of proton-nucleus reaction cross sections at two different energies or from a combination of two $\sigma_{R}$ values measured on both ${ }^{12} \mathrm{C}$ and proton targets.

The work was in part supported by JSPS KAKENHI Grants No. 24540261 and No. 25800121.
[1] L. W. Chen, C. M. Ko, B. A. Li, and J. Xu, Phys. Rev. C 82, 024321 (2010).

[2] X. Roca-Maza, M. Centelles, X. Viñas, and M. Warda, Phys. Rev. Lett. 106, 252501 (2011).

[3] M. Kortelainen, J. Erler, W. Nazarewicz, N. Birge, Y. Gao, and E. Olsen, Phys. Rev. C 88, 031305(R) (2013).

[4] T. Inakura, T. Nakatsukasa, and K. Yabana, Phys. Rev. C 88, 051305(R) (2013).

[5] P. B. Demorest, T. Pennucci, S. M. Ransom, M. S. E. Roberts, and J. W. T. Hessels, Nature 467, 1081 (2010).

[6] S. Abrahamyan et al., Phys. Rev. Lett. 108, 112502 (2012).

[7] Jefferson Lab Experiment. Proposal available at http://hallaweb.jlab.org/parity/prex.

[8] S. Terashima et al., Phys. Rev. C 77, 024317 (2008).

[9] J. Zenihiro et al., Phys. Rev. C 82, 044611 (2010).

[10] A. Tamii et al., Phys. Rev. Lett. 107, 062502 (2011).

[11] R. Kanungo et al., Phys. Rev. C 83, 021302(R) (2011).

[12] M. Takechi et al., Mod. Phys. Lett. A 25, 1878 (2010).

[13] M. Takechi (private communication); M. Fukuda, 25aXA-8, JPS annual meeting (Nishinomiya, 2012.3).

[14] D. T. Yordanov et al., Phys. Rev. Lett. 108, 042504 (2012).

[15] W. Geithner et al., Phys. Rev. Lett. 101, 252502 (2008).

[16] A. Krieger et al., Phys. Rev. Lett. 108, 142501 (2012).

[17] T. Yamaguchi et al., Phys. Rev. Lett. 107, 032502 (2011).

[18] B. Abu-Ibrahim, W. Horiuchi, A. Kohama, and Y. Suzuki, Phys. Rev. C 77, 034607 (2008).

[19] R. J. Glauber, in Lectures in Theoretical Physics, Vol. 1, edited by W. E. Brittin and L. G. Dunham (Interscience, New York, 1959), p. 315.
[20] B. Abu-Ibrahim and Y. Suzuki, Phys. Rev. C 61, 051601(R) (2000).

[21] K. Varga, S. C. Pieper, Y. Suzuki, and R. B. Wiringa, Phys. Rev. C 66, 034611 (2002).

[22] W. Horiuchi, Y. Suzuki, B. Abu-Ibrahim, and A. Kohama, Phys. Rev. C 75, 044607 (2007).

[23] W. Horiuchi and Y. Suzuki, Phys. Rev. C 74, 034311 (2006).

[24] B. Abu-Ibrahim, S. Iwasaki, W. Horiuchi, A. Kohama, and Y. Suzuki, J. Phys. Soc. Jpn. 78, 044201 (2009).

[25] W. Horiuchi, Y. Suzuki, P. Capel, and D. Baye, Phys. Rev. C 81, 024606 (2010).

[26] W. Horiuchi, T. Inakura, T. Nakatsukasa, and Y. Suzuki, Phys. Rev. C 86, 024614 (2012).

[27] J. Margeron, A. Bonaccorso, and D. M. Brink, Nucl. Phys. A 720, 337 (2003).

[28] B. Abu-Ibrahim and Y. Suzuki, Prog. Theor. Phys. 112, 1013 (2004); 114, 901 (2005).

[29] P. Capel, D. Baye, and Y. Suzuki, Phys. Rev. C 78, 054602 (2008).

[30] D. Vautherin and D. M. Brink, Phys. Rev. C 5, 626 (1972).

[31] K. T. R. Davies, H. Flocard, S. Krieger, and M. S. Weiss, Nucl. Phys. A 342, 111 (1980).

[32] J. Bartel et al., Nucl. Phys. A 386, 79 (1982).

[33] E. Chanbanat, P. Bonche, P. Haensel, J. Mayer, and R. Schaeffer, Nucl. Phys. A 627, 710 (1997).

[34] J. Belinger et al. (Particle Data Group), Phys. Rev. D 86, 010001 (2012). 